\title{
Intraperitoneal Inclusion Cyst Presenting as Intestinal Obstruction: A Clinical Dilemma
}

\author{
Amit Gupta, Utkarsh Kumar, Durga Sowmya S, Rishit Mani, Jaydeep Jain, Ashok Singh, and Sweety \\ Gupta
}

\begin{abstract}
Peritoneal inclusion cysts have been described in females of reproductive age. It is a rare cause of intestinal obstruction. Causes include pelvic inflammatory disease and prior abdominal surgery. We here present a case of young female of peritoneal inclusion cyst who presented with intestinal obstruction.
\end{abstract}

Index Terms - Peritoneum; Inclusion cyst; pelvic mass.

\section{INTRODUCTION}

Peritoneal inclusion cyst (PIC) (also known as a peritoneal pseudocyst and benign cystic mesothelioma, Entrapped ovarian cyst, Inflammatory cyst of Pelvic peritoneum occurs due to non-neoplastic reactive proliferation of mesothelial tissue. Peritoneal inclusion cysts occur in premenopausal women having history of previous abdominal or pelvic surgery, trauma, pelvic inflammatory disease, or endometriosis. It appears in relation to the peritoneum. GnRH analogs, oral contraceptives to suppress ovulation, medication for pain may be tried for conservative management. USG-guided transvaginal fluid aspiration and sclerotherapy have been attempted with less success rate. Surgical resection (recurrence rate is $30-50 \%$ ) can be done in symptomatic case. Though occurrence of metaplasia have been seen occasionally but peritoneal inclusion cysts have no malignant potential.

\section{CASE REPORT}

24 years female patient presented to surgical emergency with chief complaints of pain in lower abdomen for 1 week, insidious onset, continuous, diffuse, non-radiating, mild to moderate intensity, no aggravating and relieving factors. She also had history of abdominal distension since 3 days and non-passage of flatus and stool since 2 days. She had history of abdominal trauma 5 years back for which exploratory laparotomy was done. There was no history of fever, vomiting, jaundice, weight loss or loss of appetite. Bladder habit were normal. On physical examination there was pallor. Abdomen was distended with diffuse tenderness, shifting dullness was absent. A vertical scar mark of size $5 \mathrm{~cm}$ was present in paraumbilical area. A lump of size $20 \times 15 \mathrm{~cm}$, smooth, cystic, side to side mobility was present, well defined upper and lateral margin, lower margin not palpable involving umbilical, hypogastric, right and left lumbar and right and left iliac fossa. No hepatosplenomegaly. On percussion dullness was present over the lump. Bowel sound were absent. On digital rectal examination rectum was empty. Routine blood tests including a complete blood cell count and chemistry studies were normal. CA 125 was $30 \mathrm{U} / \mathrm{ml}$. Abdominal ultrasonography showed loculated fluid occupying whole lower abdomen and pelvis with free floating and dependent debris with bowel loops shifted upwards. CECT abdomen and pelvis reported a large well defined multi-loculated abdomino-pelvic cystic lesion measuring $10.5 \times 21.5 \times 22.5 \mathrm{~cm}$ (APxTRxCC). Lesion was displacing bilateral ovaries; however bilateral ovaries were normal in morphology. No mural nodule or calcific foci was seen within. Lesion appeared to be located in mesentery displacing the bowel loops laterally and superiorly suggestive of peritoneal inclusion cyst. (Figure 1)

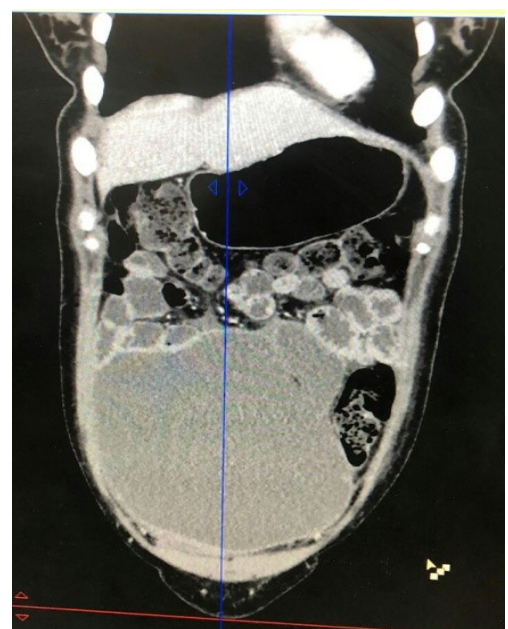

Fig.1. CECT abdomen and pelvis showing a large well defined multiloculated abdomino -pelvic cystic lesion measuring $10.5 \times 21.5 \times 22.5 \mathrm{~cm}(\mathrm{APxTR} \times C C)$.

Patient was considered for exploratory laparotomy and cyst excision. Intraoperative finding revealed cyst of size $21 \mathrm{x}$ $22 \mathrm{~cm}$ involving pelvic peritoneum densely adhered to anterior abdominal wall, bowel loops and urinary bladder. (Figure 2) 


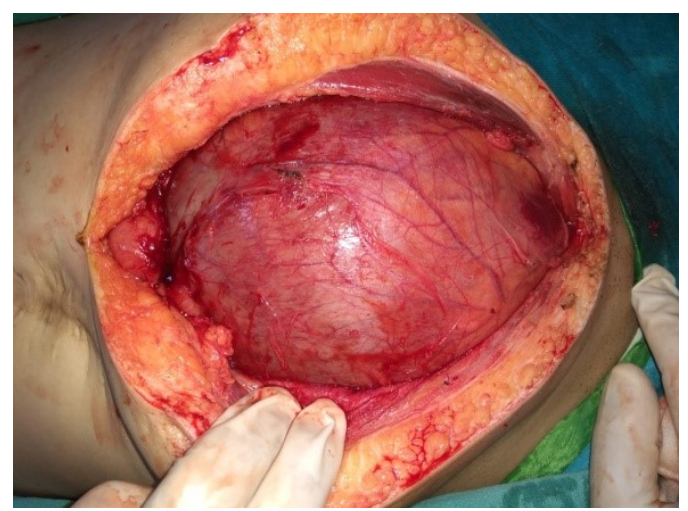

Fig. 2. Intraoperative finding showing cyst of size $21 \mathrm{x} 22 \mathrm{~cm}$ involving pelvic peritoneum densely adhered to anterior abdominal wall, bowel loops and urinary bladder.

Both ovaries were bulky. It was filled with chocolate coloured fluid. Aspirated fluid cytology examination revealed no malignant cells. On gross histopathological examination external surface was congested. Cut surface showed reddish exudates on inner surface of cyst wall with wall thickness of $5 \mathrm{~mm}$. (Figure 3)

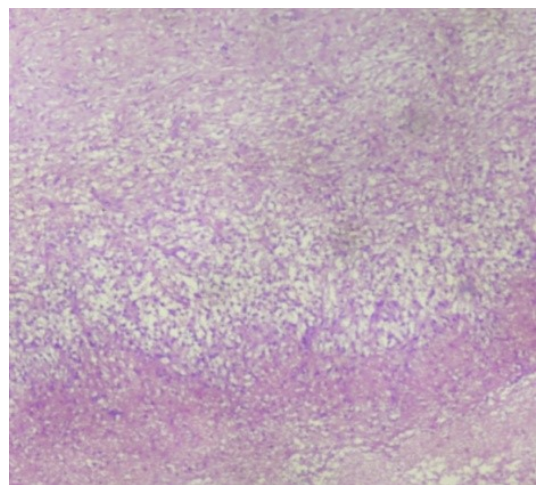

Fig:3 Microscopic section examined showed fibro collagenous cyst wall lined by inflammatory granulation tissue and necrosis. wall was edematous with congested blood vessels suggestive of pseudocyst

\section{DISCUSSION}

Peritoneum is a thin, translucent serosal membrane which is mesodermal origin that covers the surface of the peritoneal cavity. In males peritoneum is a continuous structure, resulting in a closed peritoneal cavity. In females, the peritoneum is discontinuous at the ostia of the oviducts, that lead to communication between the peritoneal cavity and extraperitoneal pelvis. Peritoneum consist of two layers: the visceral layer, which covers the surfaces of intraperitoneal organs, and the parietal layer, which covers the walls of the peritoneal cavity. It is composed of a single layer of mesothelial cells over a basal lamina; the lamina separates the mesothelial cells from a submesothelial layer of connective tissue, which consists of collagen, elastic fibres, fibroblastlike cells, arteries, veins, and lymphatics [1]. Mennemeyer and smith first described this entity in 1979 [2]. Pathogenesis of peritoneal inclusion cyst is unclear, but it revealed that Peritoneal Inclusion cyst are caused by accumulation of ovarian fluid. Normally fluid absorption from peritoneum is easy. However, absorptive capacity of the peritoneum is decreased if there is any mechanical injury, inflammation and peritoneal adhesions. Pathological basis for cyst formation is from non-neoplastic, reactive mesothelial proliferation [2]. Also growth of peritoneal inclusion cyst depends on presence of active ovary and peritoneal adhesion. Cyst is adhered to the ovary surface but does not involve ovarian parenchyma. 30\%-100\% of women had history of abdominal surgery, in our case there is also the history of abdominal trauma for which she undergoes exploratory laparotomy. Involvement of active ovaries are essential for the development of Peritoneal inclusion cyst [3]. This process typically affects female patients, with the median age at presentation being 37 years. Unlike malignant peritoneal mesothelioma, there is no association with asbestos exposure. The treatment options are controversial because it has no malignant potential. Observation, hormonal management, USG guided percutaneous drainage, USG-guided sclerotherapy and surgical excision can be planned. The surgery may vary from adhesiolysis to radical excision. Surgical excision is definitive treatment i.e. complete resection of the macroscopically visible cyst wall. There are two type of surgical approach i.e. laparoscopy or laparotomy. Recently, there are a few reports on successful laparoscopic resection of Peritoneal inclusion cyst. Porpora et al. reported that successful laparoscopic removal of a well-differentiated papillary mesothlioma of the peritoneum in a 46-year-old woman [4]. Nezhat et al. reported successful treatments of 3 cases of peritoneal mesothlioma associated with pelvic endometriosis [5]. Additionally, Kurachi et al [6] reported that after gonadotropin-releasing hormone agonist treatment, PICs became smaller. It has the potential to mimic ovarian cysts, hydrosalpinx, malignancy its nonspecific anechoic appearance [7]. A paraovarian cyst is congenital benign condition derived from the embryologic remnants of wolffian or müllerian duct.The ovary is located outside the paraovarian cyst and in case of peritoneal inclusion cysts ovary are inside the cyst or within the wall of cyst. In case of hydrosalpinx, it appears as a tubular or ovoid. Folds are often visible, and the ovary are outside of the cyst. The time interval between the insult and the detection of peritoneal inclusion cysts has ranged from 6 months to 20 years. Peritoneal inclusion cysts of upto $20 \mathrm{~cm}$ in diameter have been reported.[8] The cyst walls are mesothelial in origin, lack smooth muscle, and have usually been altered by inflammation, fibrosis, and cellular proliferation. [9]

\section{CONCLUSION}

Peritoneal inclusion cyst is a non-malignant mesothelial proliferation in reproductive females having previous surgery, trauma, pelvic infection, It has a nonspecific appearance on imaging and requires histological diagnosis. Prognosis is good after complete surgical resection since it is benign condition. Follow-up of these patients is essential to detect recurrence rate.

\section{REFERENCES}

[1] Blackburn SC, Stanton MP. Anatomy and physiology of the peritoneum. Semin. Pediatr. Surg. 2014 Dec;23(6):326-30.

[2] Mennemeyer R, Smith M. Multicystic, peritoneal mesothelioma: a report with electron microscopy of a case mimicking intra-abdominal cystic hygroma (lymphangioma) Cancer. 1979;44:692-8

[3] Peritoneal inclusion cyst | Radiology Reference Article Radiopaedia.org [Internet]. [cited 2019 Jul 24]. Available from: https://radiopaedia.org/articles/peritoneal-inclusion-cyst. 
[4] Lee SW, Lee SJ, Jang DG, Yoon JH, Kim JH. Comparision of laparoscopic and laparotomic surgery for the treatment of peritoneal inclusion cyst. Int J Med Sci. 2012;9:14-9.

[5] Porpora MG, Brancato V, D'Elia C. et al. Laparoscopic diagnosis and treatment of a well-differentiated papillary mesothelioma of the peritoneum. J Am Assoc Gynecol Laparosc. 2002;9:384-8.

[6] Nezhat FR, DeNoble SM, Brown DN. et al. Laparoscopic management of peritoneal mesothelioma associated with pelvic endometriosis. J Minim Invasive Gynecol. 2010;17:646-50.

[7] Kurachi H, Murakami T, Maeda T, et al. Value of gonadotropinreleasing hormone agonist in diagnosing peritoneal pseudocysts. Acta Obstet Gynecol Scand. 1996;75:294-297.

[8] Singh, A; Sehgal, A; Mohan, H (2015). "Multilocular peritoneal inclusion cyst mimicking an ovarian tumor: A case report". Journal of Mid-Life Health. 6 (1): 39-40.

[9] Callen PW. Ultrasonography in Obstetrics and Gynecology. 4th ed. Philadelphia: WB Saunders; 2001. pp. 871-872.

[10] Moran RE, Older RA, De Angelis GA, Baghdady BH, Chrisman HB, Ciambotti JM. Genitourinary case of the day. AJR Am J Roentgenol. $1996 ; 167: 246-251$ 